\title{
Analgesic Effect of Zolmitriptan Nanoparticles in Experimental Animal Models
}

\author{
Mr. Farshid Ali Naghi Zadeh Khezri" ${ }^{*}$ Dr. C.S.R. Lakshmi, Dr. Shachindra L. Nargund, Dr. L.V.G. Nargund, \\ Dr. Reza Alisani, Azadeh Mohammadi \\ Department of Pharmaceutics, Nargund College of pharmacy, II Main, Dattatreya Nagar, Banashankari III Stage, \\ Bangalore, Karnataka, India
}

Email: farshid_khezri @yahoo.com

\begin{abstract}
Background: Zolmitriptan is used to treat migraine and certain other headaches. This study has been done to prepare specific Zolmitriptan Nano-nasal spray (Z-NPS) by ion gelation technique to evaluate the efficacy of analgesic activity of test drug as a nano nasal spray of Zolmitriptan (Z-NPS) and conventional preparation as a standard drug (Zolmist) against Nitro-glycerine induced migraine. The study was performed on mice by using hot plate method. Methods: In this study male Swiss albino mice weighing between 25-30 grams were taken and divided into 4 groups with 6 mice in each group. Zolmitriptan loaded chitosan nanoparticles (Z-NPS) was administered nasally (using specific inhalation mask) at a dose of $5 \mathrm{mg} / \mathrm{kg}$ and was compared with the control group which received water for injection and the standard drug Zolmitriptan (Zolmist) at a same dose in mice induced migraine using hot plate method. Reaction times were measured at 10, 20, 30 and 60 minutes after drug administration. Results: The test drug (Z-NPS) at a dose of $5 \mathrm{mg} / \mathrm{kg}$ after 10 minutes of drug administration showed an increase in the reaction time compared to the standard drug (Zolmist) at same dose of drug administration. However, Z-NPS showed significant increase in the reaction time after 10 minutes as compared to Zolmist. Conclusion: Zolmitriptan has significant analgesic properties in central-analgesic model i.e. Hot-plate method. The test drug could be used for anti-migraine activity.
\end{abstract}

Keywords: Zolmitriptan, Nano-particles, Anti-migraine, Swiss albino mice, Hot-plate method, Analgesic.

\section{Background}

Migraine is a syndrome clinically manifested in attacks with dominant symptoms like unilateral, rarely generalized, recurrent headache, and occasionally lasts for days. According to different sources, it is considered that about $10-15 \%$ of world population suffers from some type of this syndrome. Migraine is most frequently clinically revealed in the age of 30-40, therefore in the most productive period with significant share in treatment costs and a great influence to the working ability of those patients. ${ }^{[1]}$

Migraine headache is a neurological disorder often initiated by a trigger and characterized by a headache, which may be accompanied by a variety of multiple organ/system symptoms, such as nausea, vomiting, and urinary frequency.

Triptans are serotonin 5-hydroxytryptamine (5-HT) receptor agonists that are generally effective, well tolerated, safe, and used to treat migraine and certain other headaches. They may be taken subcutaneously, orally as tablets, capsules, or quick-dissolving wafers, or intranasally as a spray. ${ }^{[1]}$
The use of polymeric nanoparticles (PNP) in drug delivery, generally increases the stability of the pharmaceutical agents and it can be easily and cheaply fabricated in large quantities by a multitude of methods. ${ }^{[2]}$ Nanoparticles (NPS) have been extensively investigated in biomedical and biotechnological areas and, especially, in drug delivery systems for drug targeting. The advantages of targeted drug delivery to the specific site of the body, paved the way for applying NPs to achieve this type of drug delivery. Much attention has been provided to non-parenteral routes like oral, pulmonary, nasal and opthalmic delivery of the drugs. ${ }^{[3]}$ The present study mainly aimed at delivering a drug into the brain via the intranasal route using a drug loaded chitosan nanoparticles.

The nasal route was adopted to exploit its avoidance of the hepatic first-pass metabolism to increase the absolute bioavailability.

The formulation of targeted nano nasal spray of Zolmitriptan (Z-NPS) using ion gelation technique were selected as a test drug to enhance permeability across the blood-brain barrier and therefore its brain delivery. The 
nanoparticles along with best excipients then conducted for analgesic activity using hot plate test in mice. The study was carried out with a view to elucidate the analgesic action of Z-NPS and to compare it with standard drug (Zolmist).

\section{Methods}

\section{Instrumentation and materials}

Zolmitriptan were received as a gift samples from Apotex research private limited, Bangalore. Chitosan, Nitroglycerine (NTG) and Sodium Tripoly-Phosphate (TPP) were procured from Sigma Aldrich private limited company (Pvt. Ltd). India. Phenyl ethyl alcohol was procured from Avra synthesis Pvt. Ltd. Pune, India. Polysorbate 80 (Tween 80), Sodium hydroxide, Menthol, Microcrystalline Cellulose (MCC), Glycerin, and Dextrose were purchased from S. D. Fine Chemicals Ltd. Mumbai, India.
Preparation of Zolmitriptan-loaded Chitosan Nanoparticles by ion gelation method:

Chitosan nanoparticles containing the drug Zolmitriptan (ZNPS) were prepared by the ionotropic gelation technique [5, 6]. Accurately weighed chitosan was dissolved in $1 \% \mathrm{v} / \mathrm{v}$ acetic acid solution, to which Tween 80 and the drug were added. Sodium tri poly phosphate (TPP) was dissolved in distilled water. To the chitosan-drug solution, TPP solution was added dropwise through a no.4 syringe needle and continuously stirred using a mechanical stirrer (Remi Motors- RO-123, RPM 4000) at room temperature for 30 min, which led to the formation of nanoparticles. Subsequently, the $\mathrm{pH}$ was adjusted to 5.5 with the help of a required amount of $1 \mathrm{~N}, \mathrm{NaOH}$ and then centrifuged at $12000 \mathrm{rpm}$ using a refrigerated centrifuge (Eppendorf Centrifuge 5430R).

Table 1 Experimental control factors and their levels for chitosan nanoparticles formulation

\begin{tabular}{|c|l|c|}
\hline S. No & Ingredients & Zolmitriptan \\
\hline 1 & Drug (mg/kg) & 0.5 \\
\hline 2 & Polymer (Chitosan) & $0.2 \%$ \\
\hline 3 & TPP (w/v) & $0.75 \%$ \\
\hline 4 & Tween $80(\mathrm{v} / \mathrm{v})$ & $0.25 \%$ \\
\hline 5 & Stirring speed (rpm) & 1500 \\
\hline \multicolumn{2}{|c|}{$0.1 \mathrm{~N} \mathrm{NaOH}$ is added till the $\mathrm{pH}$ of the solution reaches 5.5}
\end{tabular}

After preparation of chitosan loaded nano Zolmitriptan nasal sprays the following excipients were added and filled in metered dose nasal spray.

Tables 2 Excipients used in Zolmitriptan loaded chitosan nanoparticles nasal spray

\begin{tabular}{|c|l|l|c|}
\hline S. No & Ingredients & \multicolumn{1}{|c|}{ Function } & Percentage \\
\hline 1 & Glycerin & Humectant & $<0.5 \%$ \\
\hline 2 & MCC & Suspending agents & $<0.5 \%$ \\
\hline 3 & Phenyl ethyl alcohol & Preservative & $<0.2 \%$ \\
\hline 4 & Dextrose & Tonicity adjustment & $<0.5 \%$ \\
\hline 5 & Sodium hydroxide & pH adjustment & $<0.1 \%$ \\
\hline 6 & Menthol & Flavoring agent & $<10 \%$ \\
\hline
\end{tabular}

The prepared Zolmitriptan-loaded Chitosan nanoparticles (Z-NPS) by the Ion gelation technique were conducted for analgesic activity via intranasal drug delivery system using specific inhalation mask (Fig. 1 A) in mice.

\section{Analgesic activity characterization of Zolmitriptan Nano} nasal spray in mice using hot plate test:

The in-vivo evaluation was carried out after taking approval from the Institutional Animal Ethics Committee (IAEC) of Radiant Research, Bangalore, Karnataka, India, Reg. No. 1803/PO/RcBi/S/2015/CPCSEA.

The method validated by Galeotti $\mathrm{N}$ et $\mathrm{al}^{[7,8]}$, for evaluating antimigraine compounds was adopted.

\section{Animals}

Animals were housed under temperature $22 \pm 3$ oC, relative humidity 30-70\%, and 12-hour light and 12-hour dark cycle. Animals were housed in standard polypropylene cages with stainless steel top grill having facilities for pelleted food and drinking water in bottle. Sterile paddy husk was used as bedding material and changed every day. The diet and drinking water were free from any contaminant and provided ad libitum.

The study protocol was accepted by the institutional ethical committee and followed the guidelines of CPCSEA.

\section{Grouping}

Male Swiss albino mice, weighing 25-30 gms were divided into four groups, each group consisting of 6 animals, water for injection was used as solvent for all the groups.

The dose of test drug and standard was $0.5 \mathrm{mg} / \mathrm{Kg}$ body weight of Swiss albino mice, were dissolved in water for 


\section{International Journal of Innovative Research in Medical Science (IJIRMS) Volume 03 Issue 07 July 2018, ISSN: 2455-8737, Imp. Factor - $\underline{4.102}$ \\ Available online at $-\underline{w w w . i j i r m s . i n}$}

injection to obtain a concentration of $0.5 \mathrm{mg}$ per $0.1 \mathrm{ml}$. The groups as mentioned below (Table 3 ).

1. The first set of experiments conducted will be set as a control group. After intranasal inhalation of the water for injection by using the inhalation mask (100 $\mu \mathrm{l}$ of sample per spray), group-1 (controltreated with water for injection).

2. The second group is disease controlled group. Nitro-glycerine, dissolved in saline alcohol and propylene glycol, will be injected i.p. at a dose of 5 $\mathrm{mg} / \mathrm{kg}$ to Swiss albino mice. Systemic nitroglycerine (NTG) produces spontaneous-like migraine attacks in migraine sufferers and induces a condition of hyperalgesia in the rat $4 \mathrm{~h}$ after its administration $[9,10]$. (Disease control)

3. The third set of experiment will be conducted for determined the anti-migraine activity of Nano nasal spray of Zolmitriptan (Z-NPS) preparation (test drug) in the migraine-induced mice.

4. The fourth group is to determine the anti-migraine activity of the conventional preparation as a standard drug (Zolmist) in the migraine-induced mice.

The number of animals in each group will be six and are divided into 4 groups as follows.

Table 3 Different groups of animals used for analgesic activity

\begin{tabular}{|c|c|l|l|l|}
\hline Sl. No. & Animal No. & Group & Description & Dose \\
\hline 1 & 6 & Group I & Control (Normal) & Vehicle \\
\hline 2 & 6 & Group II & Positive Control (Nitroglycerin) & $5 \mathrm{mg} / \mathrm{kg} \mathrm{b.w}$ \\
\hline 3 & 6 & Group III & Test drug (Z-NPS) & $0.5 \mathrm{mg} / \mathrm{kg} \mathrm{b.w}$ \\
\hline 4 & 6 & Group IV & Standard drug (Zolmist) & $0.5 \mathrm{mg} / \mathrm{kg} \mathrm{b.w}$ \\
\hline
\end{tabular}

\section{Hot plate test}

\section{Procedure}

24 Male mice were divided into four groups, each group with 6 animals. Mice were randomized and grouped based on the reaction time within 10 minutes for which it was not observed a large variation by testing on four separated occasions. All the groups except control group received intraperitoneal injection of Nitroglycerine $5 \mathrm{mg} / \mathrm{kg}$ body weight $4 \mathrm{hrs}$ before the test substance administration.

Group I- Control and Group II- Positive control received vehicle, Group III and Group IV animals received $0.5 \mathrm{mg} / \mathrm{kg}$ body weight of test substance and standard drug respectively through intranasal route using specific inhalation mask (Fig. $1 \mathrm{~B})$. The hot plate test was performed in Swiss albino mice to determine the analgesic activity of test substance.

Immediately after treatment, mice were placed on hot plate maintained at $55 \pm 20 \mathrm{C}$ and the reaction time was recorded by observing the licking of the paws or jumping movements at 10,20, 30 and 60 minutes after the administration of test substance $[11,12]$. The graphical representation in figure 1 A/B.

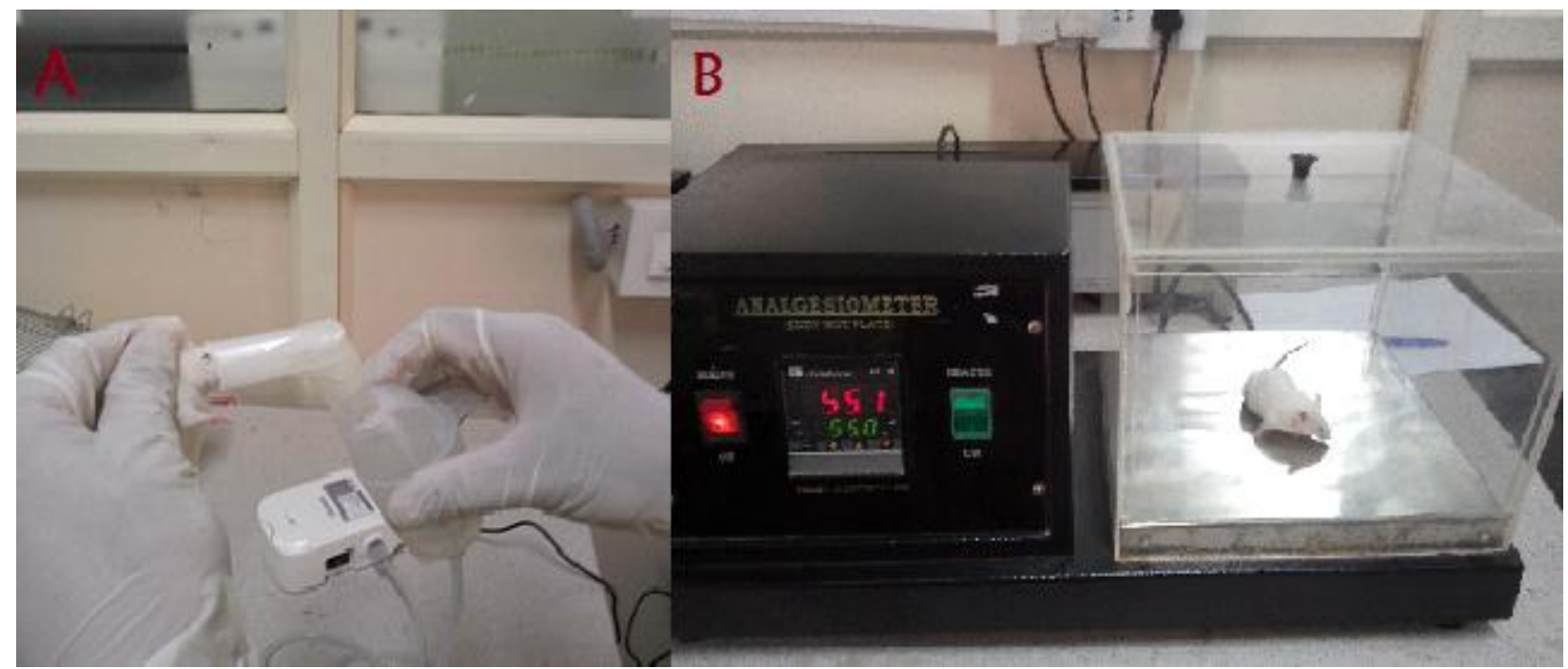

Fig. 1: Specific inhalation mask used for mice (A) and hot plate test (B)

\section{Statistical analysis}

Results were expressed as mean \pm SEM. Statistical analysis was performed by one way Anova followed by Dunnet test. Significant values were mentioned based on $\mathrm{p}$ value.
$* \mathrm{p}<0.005, * * \mathrm{p}<0.001$ and $* * * \mathrm{p}<0.0001$ when compared to positive control [13]. 


\section{Results and Discussion}

Triptan-loaded Chitosan nanoparticles was successfully formulated via the ion-gelation technique and then the formulation (Z-NPS) where conducted for analgesic activity. The results of analgesic activity of test substance (Z-NPS) using hot plate method is presented in table 4 . There was no significant difference on the thermal stimulus in mice treated with the vehicle (normal control) throughout the whole time of the experiment. Test substance administration significantly increased response time of the animals, when compared to group II. Test and standard groups showed significant analgesic activity when compared to positive control group. The results are presented in table 4 . Figure 2 demonstrated the relative activity of test substance with respect to the standard drug in the hot plate method.

Table 4: The mean reaction time to hot plate test in mice

\begin{tabular}{|c|c|c|c|c|}
\hline \multicolumn{5}{|c|}{ Latency (minutes) } \\
\hline Group & 10 Min & 20 Min & 30 Min & 60 Min \\
\hline Group I & $12.17 \pm 0.54$ & $12.17 \pm 0.79$ & $11.67 \pm 0.71$ & $10.00 \pm 0.89$ \\
\hline Group II & $50.33 \pm 2.10^{\# \# \#}$ & $45.67 \pm 2.14^{\# \# \#}$ & $41.00 \pm 1.95^{\# \# \#}$ & $36.5 \pm 1.65^{\# \# \#}$ \\
\hline Group III & $37.17 \pm 2.82^{* * * *}$ & $29.83 \pm 2.94^{* *}$ & $26.33 \pm 3.19^{* * * *}$ & $18.00 \pm 1.13^{* * * *}$ \\
\hline Group IV & $39.33 \pm 2.33^{3 * 3}$ & $31.17 \pm 4.13^{* *}$ & $27.00 \pm 2.85^{3 *}$ & $18.50 \pm 1.57^{\text {अस: }}$ \\
\hline
\end{tabular}

Values were expressed as mean \pm SEM, \#\#\#P $<0.0001$ considered significant as compared to control. $* P<0.005$, **P $<0.001$ and $* * * P<0.0001$ considered significant as compared to positive control.

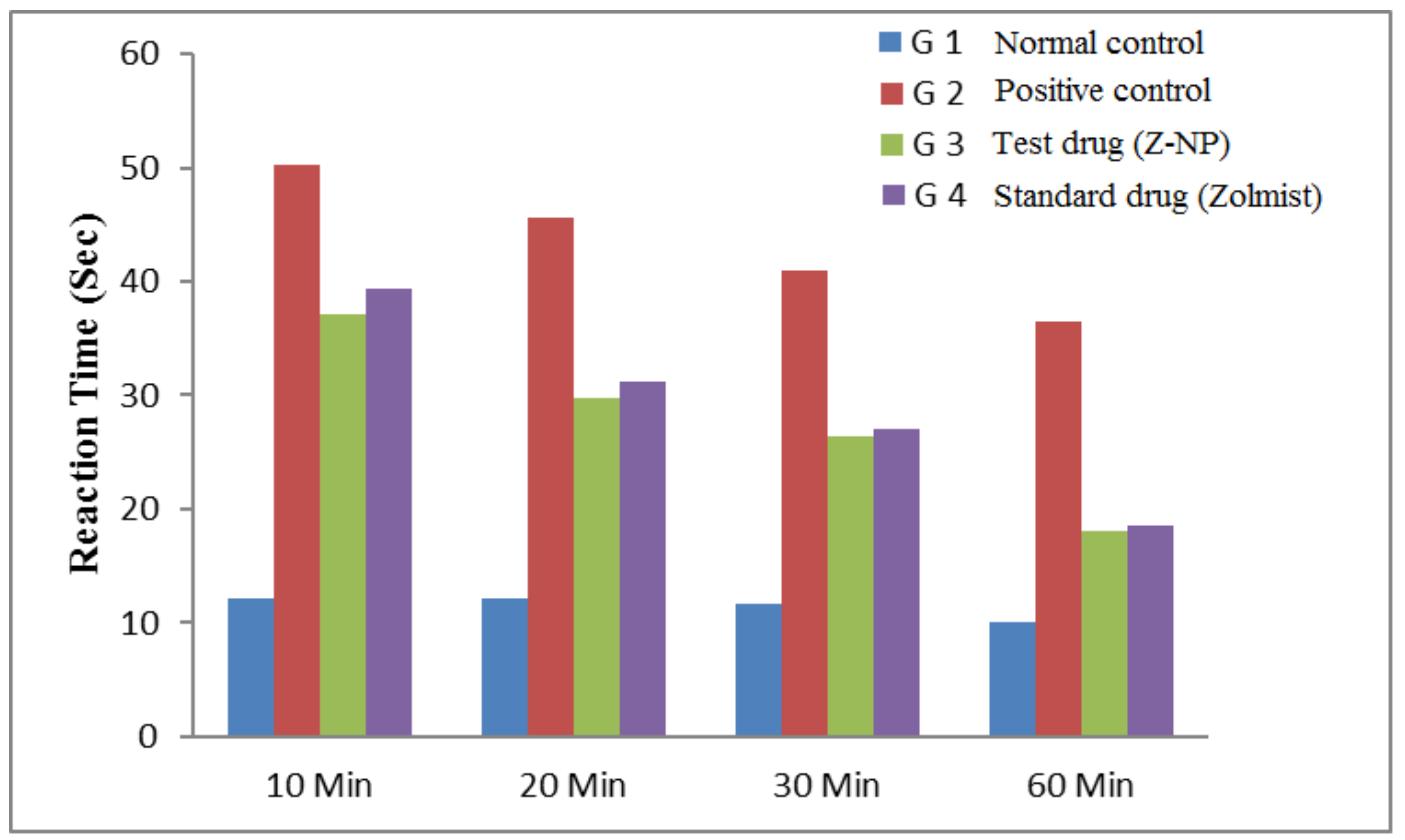

Fig. 2: Analgesic effect of test substance using hot plate method in mice. Values were expressed in Mean \pm SEM; $n=6$.

\section{Conclusion}

The results of analgesic activity by using hot plate method, indicated that the test substance, possesses significant analgesic effect at a dose of $0.5 \mathrm{mg} / \mathrm{kg}$ body weight in mice against Nitro-glycerine induced migraine in mice (Fig. 2).

\section{Abbreviations}

TPP: $\quad$ Sodium tripoly-phosphate; 5-HT: 5hydroxytryptamine; PNP: Polymeric nanoparticles; TNP: Triptan nanoparticles; NPS: Nanoparticles; MCC: Microcrystalline Cellulose; Pvt-Ltd: privet limited company; $\mathrm{NaOH}$ : Sodium hydroxide; TNNS: Triptan Nano Nasal Spray; RPM: Revolutions per minute; N: Normality; VS: Versus; NM: Nanometer; Z-NPS; Zolmitriptan-loaded Chitosan Nanoparticles.

\section{Acknowledgements}

Authors very thankful to Apotex Research Private Limited, Bangalore for providing the gift samples of Triptans. We thank Radiant Research of Science (RRS) Bangalore, Vision Group Science and Technology, Government of Karnataka and the management and Principal, Nargund College of Pharmacy, Bangalore, Karnataka, India, for their immense support in accomplishing the work.

\section{Funding}

This research was part of $\mathrm{Ph} . \mathrm{D}$. thesis of the first author and was performed using equipment's purchased under Vision Group of Science and Technology at Nargund College of pharmacy affiliated to Rajiv University of Health Sciences, Karnataka, Bangalore. 
Availability of data and materials

All data generated or analyzed during this study are included in this published article.

\section{Authors' contributions}

FA participated in design of the study, carried out all experiments and drafted the manuscript. LCSR participated in design of the study, interpretation and analysis of data and major contributor in writing the manuscript. LVG Principal Nargund College of pharmacy, Bangalore, India, participated in management and immense support in accomplishing the studies. All authors read and approved the final manuscript.

\section{Competing interests}

The authors declare that they have no competing interests.

\section{Consent for publication}

Not applicable.

Ethics approval and consent to participate

Radiant Research, Bangalore, Karnataka, India.

Registration. No. 1803/PO/RcBi/S/2015/CPCSEA.

\section{Publisher's Note}

Springer Nature remains neutral with regard to jurisdictional claims in published maps and institutional affiliations.

\section{References}

[1] Kassem A, Labib GS (2017) Flash dissolving sublingual almotriptan malate lyotabs for management of migraine. Int $\mathbf{J}$ Pharm Pharm Sci 9:125-31.

[2] Mark H, Kim P (2009) Drug Class Review Triptans. Portland, OR: Oregon Health and Science University 4: 5-6.

[3] Mistry A, Stolnik S, Illum L (2009) Nanoparticles for direct nose-to-brain delivery of drugs. Int $\mathrm{J}$ Pharm 379 (1): 146-57.

[4] Lupin RP (2016) Formulation and Product Development of Nasal Spray: An Overview Journal of Applied Medical Sciences 4 (8): 2976-85.

[5] Tiyaboonchai W (2003) Department of Pharmaceutical Technology, Faculty of Pharmaceutical Sciences. Naresuan University Journal 11 (3): 51-66

[6] Mahapatro A, Singh DK (2011) Biodegradable nanoparticles are excellent vehicle for site directed in-vivo delivery of drugs and vaccines. Journal of Nanobiotechnology 9: 1-55.

[7] Galeotti N, Ghelardini C, Grazioli I, Uslenghi C (2002) Indomethacin, caffeine and prochlorperazine alone and combined revert hyperalgesia in in-vivo models of migraine. Pharmacology research 46 (3): 245-50.
[8] Jovicic A, Raicivic and Boskovic B (2013) Analgesic efficacy of famalgin and Imigran in patients with acute Migraine attack. International journal of pharmaceutical and chemical sciences 2 (2): 255-61.

[9] Rosaria G, Antonina S, Giorgio S, Mauro M, Giuseppe N, Cristina T (2014) Activation of CB2 receptors as a potential therapeutic target for migraine: evaluation in an animal model. Journal of Headache and Pain 15 (14): 2-8.

[10] Yuanchao Li, Qin Zhang, Dandan Qi, Li Zhang, Lian Yi, Qianqian Li and Zhongling Zhang (2016) Valproate ameliorates nitroglycerin-induced migraine in trigeminal nucleus caudalis in rats through inhibition of NF-кB. The Journal of Headache and Pain 17 (49): 1-9.

[11] Malmberg AB, Bannon AW (1999) Models of nociception: hot-plate, tail-flick, and formalin tests in rodents. Current Protocols in Neuroscience 8 (9): $1-15$.

[12] Patil B, Ashok B, Kakkeri RH, Shrinivas R, Basavambika A (2013) A comparative study of analgesic activity of fluoxetine with ibuprofen and pentazocine in rodent models. Journal of Evolution of Medical and Dental Sciences 2 (33): 62-9.

[13] Team RC (2014) A language and environment for statistical computing. Foundation for Statistical Computing, Vienna, Austria. ISBN 3 (9): 51-7. 